\title{
WCDMA downlink capacity of cigar-shaped microcells using soft hand-over with SIR-based power control for over-ground train service
}

\author{
Bazil Taha Ahmed , Miguel Calvo Ramon \\ Universidad Autónoma de Madrid, Escuela Politecnica Superior, Madrid, 28049, Spain \\ Universidad Politécnica de Madrid, ETSI Telecomunicación, Madrid, 28040, Spain
}

\begin{abstract}
In this paper, the downlink sector capacity of a cigar-shaped microcells using wideband code-division multiple-access (WCDMA) with soft hand-over (SHO) mode is analyzed. The two-slope propagation loss with log-normal shadowing is used in the analysis where a model of eight cigar-shaped microcells is utilized to calculate the downlink sector capacity. The performance of the downlink is studied for different sector radii, standard deviations of the shadowing and propagation exponents. It is found that, for a sector range higher than $940 \mathrm{~m}$, increasing the sector range will reduce the downlink sector capacity. Also it is found that increasing the value of the propagation parameters will reduce the downlink sector capacity. In many cases, the downlink sector capacity will be code limited since the theoretical downlink sector capacity is higher than the number of codes assigned to each sector. The high theoretical downlink sector capacity is due to the low value $(0.06-0.1)$ of the WCDMA orthogonality factor of rural zone microcells.
\end{abstract}

Keywords: WCDMA; Downlink capacity; Shadowing; Soft hand-over (SHO) margin

\section{Introduction}

CDMA cellular systems including WCDMA for UMTS employs an elaborated hand-over method in which a mobile station is simultaneously connected to several base stations. This method of hand-over is called soft hand-over ( $\mathrm{SHO}$ ). CDMA techniques make possible to maintain an old connection while adding a new one (make before break); however, additional resources (codes) from several base stations are required In downlink, this is achieved by multiple site transmission, which implies that several base stations transmit the same signal to a certain mobile station (MS).

The capacity in WCDMA systems is commonly limited by interference. In order to reduce the interference level in downlink, power control techniques are proposed. Using a power control algorithm that is based on signal to interference ratio (SIR) in downlink, the power that is transmitted to the MS is adjusted to achieve the energy-per-bit to noise power spectral density ratio $\left(E_{\mathrm{b}} / N_{\mathrm{o}}\right)$ requirements Therefore, more capacity can be achieved by the system if SIR-based power control techniques are used since system interferences are reduced

Two issues must be taken into account in SHO performance evaluation, namely

- The active set where usual number of the involved base stations is $2-3$.

- The SHO margin $\left(M_{\mathrm{SH}}\right)$ with a typical value of 3-6 dB.

The active set is the group of base stations to which a user terminal is connected. $M_{\mathrm{SH}}$ is the maximum allowed difference (measured in decibels) between the power that is received from the best server base stations and the power received from a candidate base station that is included in the active set of the user terminal

In it has been shown that SHO used in the uplink of the Mobile Telecommunications System (UMTS), reduces 
interference; therefore, SIR is increased. This effect is modelled as a SHO gain and can be used to offer higher quality services to users or to allocate a higher number of users in a cell (higher capacity). These analyses have demonstrated that capacity in uplink is always increased with $M_{\mathrm{SH}}$. In

Mehailescu et al. analyzed the downlink SHO performance in terms of $E_{\mathrm{b}} / N_{\mathrm{o}}$, assuming two candidate base stations in the active set. Connection probabilities were calculated to determine the connection situation of each mobile station location. SIR as a function of the distance to the interference base station has been given for different scenarios.

The effect of SHO on power-controlled downlink systems has been studied Yang et al. studied the effect of cross-correlated shadowing on the SIR using hard hand-over and SHO algorithms. It has been demonstrated that the constant cross-correlation model overestimates the SIR in WCDMA systems. In Zhang et al. obtained the downlink capacity gain for SHO CDMA systems by dividing a hexagonal layout in defined connection zones. In the performance of hard and SHO algorithms in a downlink WCDMA system using a probabilistic method has been studied. The mean active set number (the average number of base stations in the active set) is calculated in a mobility scenario where the environment model is limited to the path between two base stations.

The uplink capacity of the cigar-shaped microcells in highways has been studied the uplink capacity of WCDMA system used to service trains has been investigated. Downlink capacity has been studied for the case of street microcells assuming that omnidirectional antennas are used in the base stations without the use of the power control technique

We have to mention that, power control is efficient in increasing the downlink capacity if users are uniformly distributed within the cell. This means that power control is used to mitigate the near-far problem. Thus, for a cell with all users near to the cell border, power control is not efficient since all users are affected by quasi the same interference power.

It is well known that urban microcell shapes may approximately follow the street pattern and that it is possible to have cigar-shaped microcells. Cigar-shaped microcells are used to have coverage in tunnels, groove-ways, highways and streets.

The conditions that describe the rural highway cigarshaped microcells under this study are:

- The number of directional sectors of the cigar-shaped microcell is two and a directional antenna is used in each sector.

- The microcell sector has typically a range of $1 \mathrm{~km}$.

- User speed can reach $120 \mathrm{~km} / \mathrm{h}$.

The main contribution of this work is the calculation of the WCDMA downlink capacity of cigar-shaped microcells using soft hand-over with SIR-based power control when all users exist within a limited space of the microcell (only within train) a special case not studied before for which power control is not efficient.

The rest of the paper has been organized as follows. In Section 2, the propagation model is given. In Section 3, the downlink interference is analyzed. Numerical results are presented in Section 4. Finally, in Section 5 conclusions are drawn.

\section{Propagation model}

it has been found that the two-slope propagation model is the most appropriate model of propagation to be used in the analysis of the WCDMA capacity of microcells in rural zones. In the first slope region of the model, waves propagate in the quasi free space mode $\left(s_{1} \approx 2\right)$ while in the second slope region the propagation exponent $s_{2}$ is higher with value that can reach 5 . Change of slope from $s_{1}$ to $s_{2}$ happens at the break point.

For the above mentioned reason, the two-slope propagation model with log-normal shadowing will be used in the analysis of the downlink. In this way the path loss is given by:

$$
\begin{aligned}
& L_{\mathrm{p}}(\mathrm{dB}) \approx L_{\mathrm{b}}+10_{s_{1}} \log _{10}\left(\frac{r}{R_{\mathrm{b}}}\right)+L_{\mathrm{g}}+\xi_{1} \quad \text { If } r \leqslant R_{\mathrm{b}} \\
& L_{\mathrm{p}}(\mathrm{dB}) \approx L_{\mathrm{b}}+10_{s_{2}} \log _{10}\left(\frac{r}{R_{\mathrm{b}}}\right)+L_{\mathrm{g}}+\xi_{2} \quad \text { If } r>R_{\mathrm{b}}
\end{aligned}
$$

where $L_{\mathrm{g}}$ is the car's window penetration loss, $r$ is the disance between the microcell base station and the mobile, $L_{\mathrm{b}}$ (propagation loss at the break point) is given as:

$L_{\mathrm{b}}(\mathrm{dB})=20 \log _{10}\left(\frac{4 \pi}{\lambda}\right)+10_{s_{1}} \log _{10}\left(R_{\mathrm{b}}\right)$

The break-point distance $R_{\mathrm{b}}$ is given by Tsai and Chang [12]:

$R_{\mathrm{b}} \approx \frac{4 h_{\mathrm{b}} h_{\mathrm{m}}}{\lambda}$

where

- $h_{\mathrm{b}}$ is the base station antenna height,

- $h_{\mathrm{m}}$ is the mobile antenna height,

- $\lambda$ is the wavelength and

- $\xi_{1}$ and $\xi_{2}$ are Gaussian random variables of zero-mean and a standard deviation of $\sigma_{1}$ and $\sigma_{2}$, respectively, representing the shadowing effect (path loss deviation from the average value).

Typical values of the above mentioned parameters are:

- $s_{1}=2.00-2.25$,

- $s_{2}=4.0-4.5$,

- $\sigma_{1}=2.0-3.0 \mathrm{~dB}$,

- $\sigma_{2}=4.0-6.0 \mathrm{~dB}$,

- $R_{\mathrm{b}}=300 \mathrm{~m}$,

- $L_{\mathrm{g}}=3 \mathrm{~dB}$. 


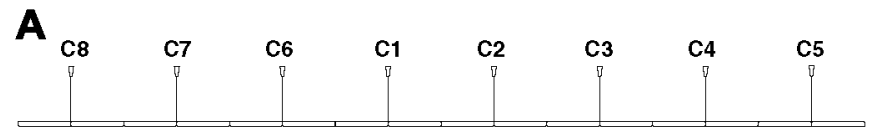

\section{B}

Left sector coverage

Right sector coverage

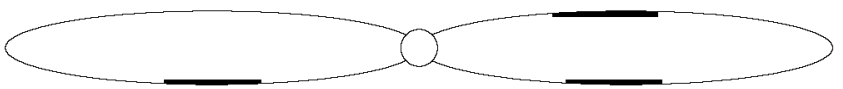

Fig. 1. The eight microcells model and the microcell coverage. (A) The eight microcells model. (B) The microcell coverage.

\section{Downlink interference analysis}

Fig. 1 shows the configuration of the eight microcells model and the coverage of each microcell. The microcells are assumed to be are regularly spaced every $2 R$. Thus, the sector range is $R$. The downlink sector capacity will be studied considering the right sector of the microcell $\mathrm{C} 1$. If the user $i$ is at a distance $r_{\mathrm{io}}$ from the microcell base station under study $(\mathrm{C} 1)$ and at a distance $r_{\text {id }}$ from the interfering microcell base station $d$ as shown in Fig. 2, then the ratio of the interference signal ratio $L\left(r_{\mathrm{id}}, r_{\mathrm{io}}\right)$ due to the distance only is given as:

- If $\left(r_{\mathrm{id}}\right.$ and $\left.r_{\mathrm{io}} \leqslant R_{\mathrm{b}}\right)$ then $L\left(r_{\mathrm{id}}, r_{\mathrm{io}}\right)$ is:

$$
L\left(r_{\mathrm{id}}, r_{\mathrm{io}}\right)=\left(r_{\mathrm{io}} / r_{\mathrm{id}}\right)^{s_{1}}
$$

- If $r_{\mathrm{id}}>R_{\mathrm{b}}$ and $r_{\mathrm{io}} \leqslant R_{\mathrm{b}}$ then $L\left(r_{\mathrm{id}}, r_{\mathrm{io}}\right)$ is given as:

$$
L\left(r_{\mathrm{id}}, r_{\mathrm{io}}\right)=r_{\mathrm{io}}^{s_{1}} /\left\{R_{\mathrm{b}}^{s_{1}}\left(r_{\mathrm{id}} / R_{\mathrm{b}}\right)^{s_{2}}\right\}
$$

- If $\left(r_{\mathrm{id}}\right.$ and $\left.r_{\mathrm{io}}>R_{\mathrm{b}}\right)$ then $L\left(r_{\mathrm{id}}, r_{\mathrm{io}}\right)$ is:

$$
L\left(r_{\text {id }}, r_{\text {io }}\right)=\left(r_{\text {io }}, r_{\text {id }}\right)^{s_{2}}
$$

Now the ratio of the interference signal $L_{\mathrm{shd}}\left(r_{\mathrm{id}}, r_{\mathrm{io}}\right)$ due to the distance and shadowing is given by

$L_{\text {shd }}\left(r_{\text {id }}, r_{\text {io }}\right)=10^{\left(\xi_{\text {id }}-\xi_{\text {io }}\right) / 10} L\left(r_{\text {id }}, r_{\text {io }}\right)$

$\xi_{\text {id }}$ and $\xi_{\text {im }}$ are given as

- In case of $\left(r_{\mathrm{id}}\right.$ and $\left.r_{\mathrm{io}} \leqslant R_{\mathrm{b}}\right)$ then $\xi_{\mathrm{id}}=\xi_{1}$ and $\xi_{\mathrm{io}}=\xi_{1}$.

- If $r_{\text {id }}>R_{\mathrm{b}}$ and $r_{\text {io }} \leqslant R_{\mathrm{b}}$ then $\xi_{\text {id }}=\xi_{2}$ and $\xi_{\text {io }}=\xi_{1}$.

- In case of $\left(r_{\mathrm{id}}\right.$ and $\left.r_{\mathrm{io}}>R_{\mathrm{b}}\right)$ then $\xi_{\mathrm{id}}=\xi_{2}$ and $\xi_{\mathrm{io}}=\xi_{2}$.

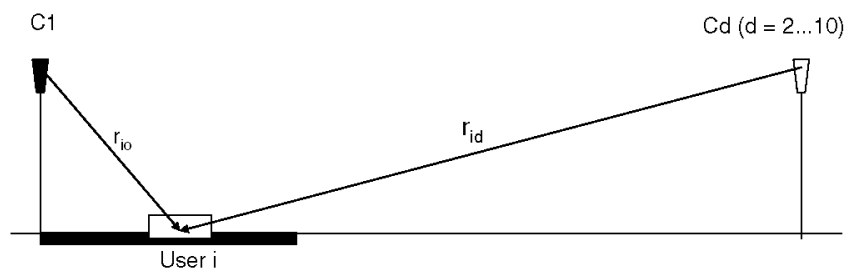

Fig. 2. Schematic diagram of microcells base stations and mobiles for rural zones microcells.
To calculate the mean value of the downlink capacity it is necessary to calculate the expected value of the intracellular interference to the desired value ratio, the expected value of the intercellular interference to the desired value ratio and the expected value of the thermal noise to the desired value ratio.

For the user under study, the intercellular interference is due to the rest of the base stations that it is not connected with. The expected value of the intercellular interference power to the desired signal power $E\left[\frac{I_{\text {inter }}}{C}\right]$ when the signals emitted from $\mathrm{C} 2$ are interfering signals is given by Mehailescu

$$
\begin{aligned}
E\left[\frac{I_{\text {inter }}}{C}\right]= & 0.5 \frac{p_{t}}{p_{1, t}} \mathrm{e}^{\left(\beta^{2} \sigma^{2} / 2\right)} \sum_{d=2}^{8} L\left(r_{\text {id }}, r_{\mathrm{io}}\right) Q \\
& \times\left[\beta \sigma+\frac{10 \log _{10}\left\{L\left(r_{\mathrm{id}}, r_{\mathrm{io}}\right)\right\}+M_{\mathrm{SH}}}{\sigma}\right]
\end{aligned}
$$

where

- The factor 0.5 is due to the fact that half of the transmitted power will be transmitted in the direction of the user under study due to the utilization of two directive antennas in each base station.

- $C$ is the desired signal power,

- $p_{\mathrm{t}}$ is the base station total transmitted power in two directions using two directive antennas at each base stations.

- $p_{1, i}$ is the power transmitted by the base station under study to the user at the location $i$.

- $\beta=(\ln 10) / 10$,

- $M_{\mathrm{SH}}$ is the soft hand-over margin (dB) and

- $\sigma$ is standard deviation of $\left(\xi_{\text {id }}-\xi_{\text {im }}\right)$.

- $Q$ is the Gaussian $Q$ function given by:

$$
Q(x)=\frac{1}{\sqrt{2 \pi}} \int_{x}^{\infty} \mathrm{e}^{-y^{2} / 2} \mathrm{~d} y
$$

Now the general value of $\sigma^{2}$ is given as:

- When $\left(r_{\mathrm{id}}\right.$ and $\left.r_{\mathrm{io}} \leqslant R_{\mathrm{b}}\right)$ then $\sigma_{\mathrm{id}}=\sigma_{1}$, also $\sigma_{\mathrm{io}}=\sigma_{1}$ then

$$
\sigma^{2}=2\left(1-C_{\mathrm{do}}\right) \sigma_{1}^{2}
$$

where $C_{\mathrm{do}}$ is the inter-sites correlation coefficient.

- If $r_{\mathrm{id}}>R_{\mathrm{b}}$ and $r_{\mathrm{id}} \leqslant R_{\mathrm{b}}$ then the value of $\sigma^{2}$ is given by

$$
\sigma^{2}=\left(\sigma_{1}-\sigma_{2}\right)^{2}+2\left(1-C_{\mathrm{do}}\right) \sigma_{1} \sigma_{2}
$$

- When $\left(r_{\mathrm{id}}\right.$ and $r_{\mathrm{io}}>R_{\mathrm{b}}$ ) then $\sigma_{\mathrm{id}}=\sigma_{2,}$, also $\sigma_{\mathrm{io}}=\sigma_{2}$ then

$$
\sigma^{2}=2\left(1-C_{\mathrm{do}}\right) \sigma_{2}^{2}
$$

For the user under study, the intracellular interference is due to the sum of the received power of the rest of the users of the same microcell under study and the received power of the pilot signal taking into account the effect of the orthogonality factor. The expected value of the intracellu- 
lar interference power to the desired signal power ratio due to base station 1 is given by:

$E\left[\frac{I_{\text {intra }}}{C}\right]=0.5 \delta\left(\frac{p_{t}}{p_{1, i}}-1\right)$

where $\delta$ is the orthogonality factor of the order of 0.1 .

To calculate the desired signal power to the noise ratio, it is necessary to calculate the expected value of the received signal power using the appropriate model of propagation. The expected value of the desired signal power to the noise ratio is given by:

$E\left[\frac{N_{o}}{C}\right]=\frac{N_{o}}{p_{1, i} 10^{-\left(L_{1, l}(\mathrm{~dB})-G_{\mathrm{TX}}(\mathrm{dB})-G_{\mathrm{RX}}(\mathrm{dB})\right) 10}}$

where $L_{1, i}$ is the path loss between the base station 1 and the location $i$ measured in $\mathrm{dB}, G_{\mathrm{TX}}$ is the base station antenna gain measured in $\mathrm{dB}$ and $G_{\mathrm{RX}}$ is the mobile antenna gain assumed to be $0 \mathrm{~dB}$. In $L_{1, i}$ the effects of the distance and shadowing are included assuming $\xi_{2}$ to be $2.33 \sigma_{2}$.

When the power control is inhibited, the power assigned to each user is the user's total transmitted power divided uniformly between all of them taking into account the effect of the user activity factor $\alpha$. Thus, the power transmitted from the base station to the desired user at location $i$ is given by:

$p_{1, l} \approx \frac{0.5 p_{t} p_{\mathrm{ch}}}{N_{u} \alpha}$

where

- $p_{\mathrm{ch}}$ is the ratio of the power assigned to all of the users $\approx 0.8$ of the base station total transmitted power $(0.2$ of the base station total transmitted power is assigned to the pilot and common channel),

- $N_{\mathrm{u}}$ is the number of the users uniformly distributed within the microcell sector and

- $\alpha$ is the source activity factor $(0.66$ for voice and it is 1 for data).

Calculating the expected value of the intracellular interference to the desired value ratio, the expected value of the intercellular interference to the desired value ratio and the expected value of the thermal noise to the desired value ratio it is possible to calculate the signal to interference ratio. The ratio $C / I$ of the user under consideration is given by:

$C / I=\frac{1}{E\left[\frac{\left.I_{\text {intra }}\right]}{C}\right]+E\left[\frac{I_{\text {inter }}}{C}\right]+E\left[\frac{N_{o}}{C}\right]}$

In WCDMA the energy-per-bit to the noise power $\left(E_{\mathrm{b}} / N_{\mathrm{o}}\right)$ ratio is given by:

$E_{\mathrm{b}} / N_{o}=G_{\mathrm{p}}(C / I)$

where $G_{\mathrm{p}}$ is the WCDMA processing gain.

Finally, the maximum number of users ( $N_{\mathrm{ui}}$ ) that can be supported at the location $i$ can be found using Eq. (18) increasing the number of the users within the sector and calculating $E_{\mathrm{b}} / N_{\mathrm{o}}$. The process will be stopped when the value of $E_{\mathrm{b}} / N_{\mathrm{o}}$ is little bit greater than the required $E_{\mathrm{b}} / N_{\mathrm{o}}$.

When perfect power control is used, then, the downlink capacity of the sector is average value of the capacity in all point where users exists. Thus, the downlink capacity is given by:

$N_{\mathrm{pc}} \approx \operatorname{avg}\left(N_{\text {ui }}\right) \mid i=i_{o} \rightarrow i_{o}+L$

where $L$ is the train length.

\section{Numerical results}

For our calculations some reasonable figures are applied. The inter-sites correlation coefficients $C_{\mathrm{do}}=0.5$, $\delta=0.1, s_{1}=2, s_{2}=4, \sigma_{1}=3 \mathrm{~dB}, \sigma_{2}=6 \mathrm{~dB}, R_{\mathrm{b}}=300 \mathrm{~m}$, $R=1000 \mathrm{~m}$ and $L=80 \mathrm{~m}$ unless other values are mentioned. Also we assume that the base station total transmitted power is $1 \mathrm{~W}$, mobile receiver thermal noise power of $-100 \mathrm{dBm}$, directive antenna gain of $10 \mathrm{~dB}$ and a frequency of operation of $2.14 \mathrm{GHz}$.

Since users exist only within the train, then, the calculation of the interference and capacity is done only for the zone where the train exist, i.e., not in all the points of the sector under study. For this reason we will get two extreme cases. The first one which is the worst case will happen when the trains are at the middle of the distance between the base stations. In this case the interference is the highest possible and for that the downlink capacity is the minimum. The second case which is the best case will happen when the trains are very near to the base stations. In this case the total interference is the lowest possible. Thus, the downlink capacity is the maximum possible.

First we study the case of voice service assuming that $\alpha=0.66, G_{\mathrm{p}}=256$ and $\left(E_{\mathrm{b}} / N_{\mathrm{o}}\right)_{\mathrm{req}}=7 \mathrm{~dB}$. In this case, the maximum number of codes assigned for the sector is

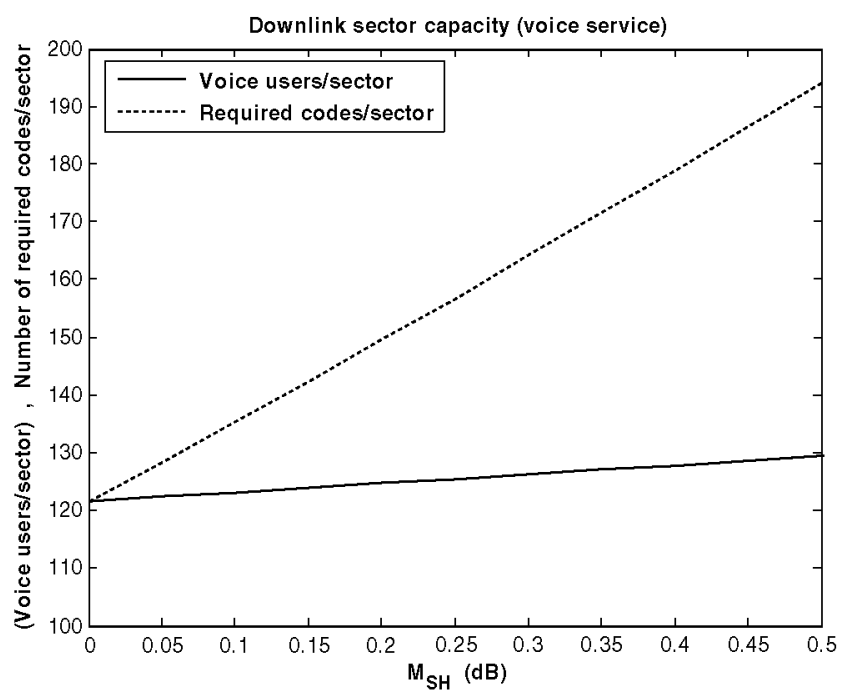

Fig. 3. Worst case sector downlink capacity and the required codes/sector as a function of $M_{\mathrm{SH}}$ for the standard parameters. 


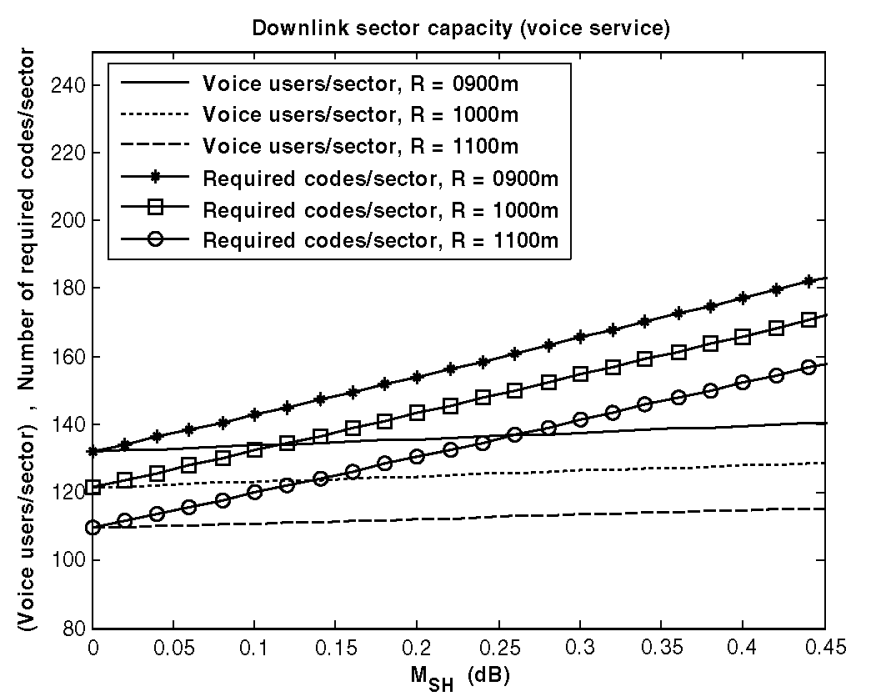

Fig. 4. Worst case sector downlink capacity and the required codes/sector as a function of $M_{\mathrm{SH}}$ for different sector range $R$.

$128\left(G_{\mathrm{p}} / 2\right)$ codes. Fig. 3 shows the worst case sector downlink capacity as a function of $M_{\mathrm{SH}}$. It can be noticed that at $M_{\mathrm{SH}}$ of $0.047 \mathrm{~dB}$, the sector capacity is 122 voice users and that the required number of codes is 128 codes. In this case six extra codes are used for the soft hand-over algorithm. To calculate the sector downlink capacity we have to define the $M_{\mathrm{SH}}$ such that the required codes are $G_{\mathrm{p}} / 2$. The sector downlink capacity is the number of users at the previously defined $M_{\mathrm{SH}}$.

Fig. 4 depicts the worst case sector downlink capacity as a function of $M_{\mathrm{SH}}$ for three values of the sector range $R$. The sector downlink capacity is $(+128,122$ and 111$)$ voice users for a sector range of $(900,1000$ and 1100$) \mathrm{m}$, respectively. To get the maximum possible sector downlink capacity, the sector range should be up to $940 \mathrm{~m}$. The practical sector range could be $1 \mathrm{~km}$.

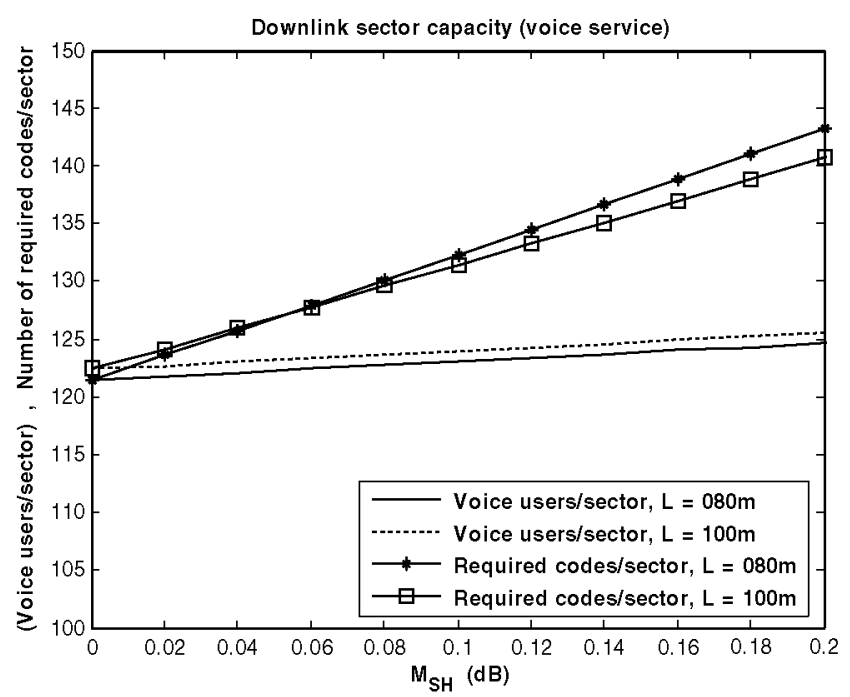

Fig. 5. Worst case sector downlink capacity and the required codes/sector as a function of $M_{\mathrm{SH}}$ for different train length $L$.
Fig. 5 portrays the worst case sector downlink capacity as a function of $M_{\mathrm{SH}}$ for two values of the train length $L$. The sector downlink capacity is (122 and 123) voice users for train length of (80 and 100) $\mathrm{m}$, respectively. Thus, it can be noticed that the effect of the train length is very little.

Fig. 6 represents the worst case sector downlink capacity as a function of $M_{\mathrm{SH}}$ for two values of $s_{1}$. The sector downlink capacity is (122 and 75) voice users for $s_{1}$ of 2.00 and 2.25 , respectively. Thus, it can be noticed that for higher $s_{1}$, the sector downlink capacity will be lower.

Fig. 7 shows the worst case sector downlink capacity as a function of $M_{\mathrm{SH}}$ for two values of $s_{2}$. The sector downlink capacity is (122 and 110) voice users for $s_{2}$ of 4.0

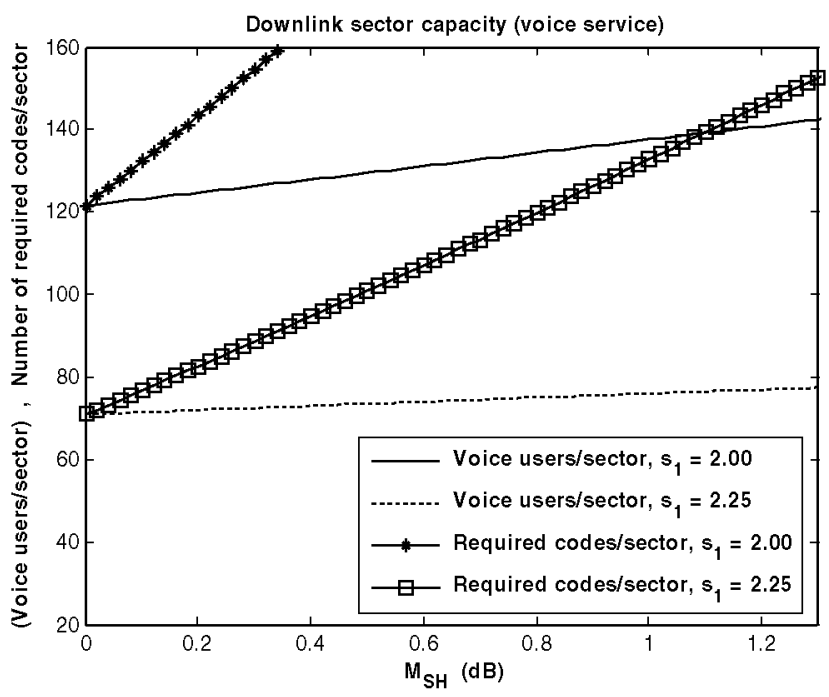

Fig. 6. Worst case sector downlink capacity and the required codes/sector as a function of $M_{\mathrm{SH}}$ for different values of $s_{1}$.

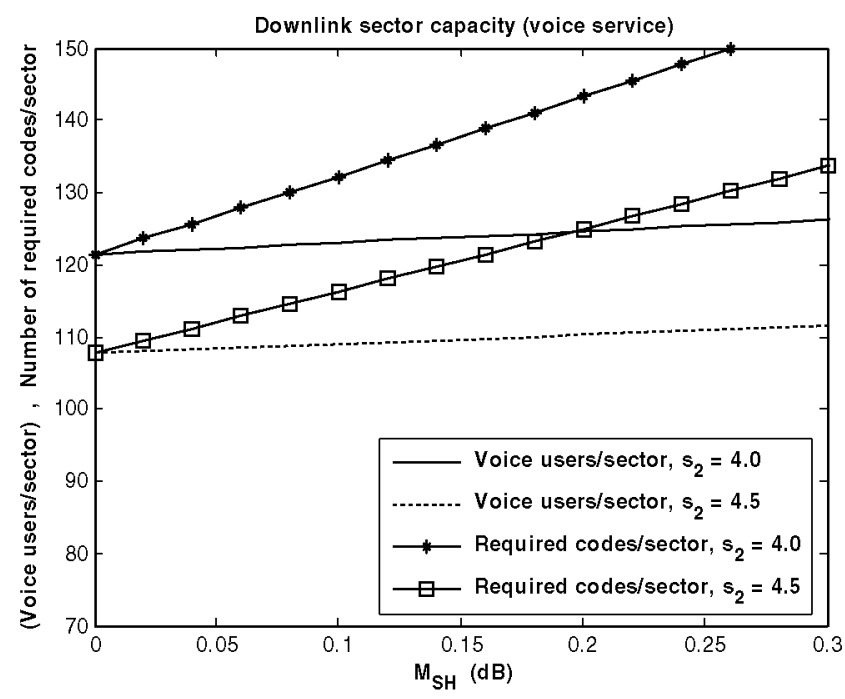

Fig. 7. Worst case sector downlink capacity and the required codes/sector as a function of $M_{\mathrm{SH}}$ for different values of $s_{2}$. 


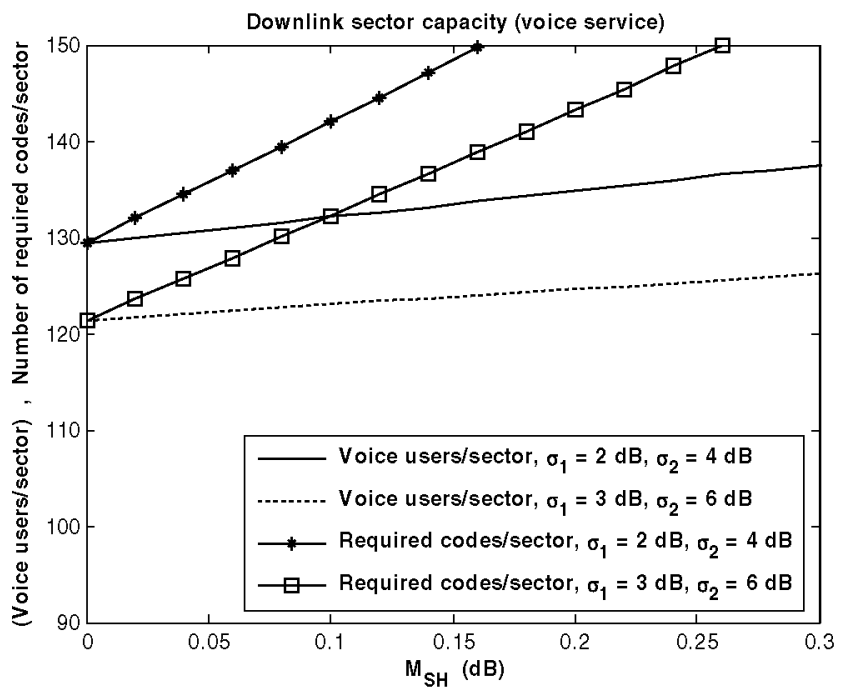

Fig. 8. Worst case sector downlink capacity and the required codes/sector as a function of $M_{\mathrm{SH}}$ for different values of $\sigma_{1}$ and $\sigma_{2}$.

and 4.5 , respectively. Thus, it can be noticed that for higher $s_{2}$, the sector downlink capacity will be lower.

Fig. 8 depicts the worst case sector downlink capacity as a function of $M_{\mathrm{SH}}$ for different values of value of $\sigma_{1}$ and $\sigma_{2}$. The sector downlink capacity is $(+128$ and 122$)$ voice users for $\left(\sigma_{1}=2 \mathrm{~dB}, \sigma_{2}=4 \mathrm{~dB}\right)$ and $\left(\sigma_{1}=3 \mathrm{~dB}, \sigma_{2}=6 \mathrm{~dB}\right)$, respectively. Thus, it can be noticed that for higher $\sigma_{1}$ and $\sigma_{2}$, the sector downlink capacity will be lower.

Fig. 9 represents the worst case sector downlink capacity as a function of $M_{\mathrm{SH}}$ for two different values of the orthogonality factor $\delta$. The sector downlink capacity is $(+128$ and 122 ) voice users for an orthogonality factor $\delta$ with a value of $(0.06$ and 0.10$)$, respectively. Thus, it can be noticed that for higher $\delta$, the sector downlink capacity will be lower. This is due to fact that, higher $\delta$ give a rise to higher intracellular interference.

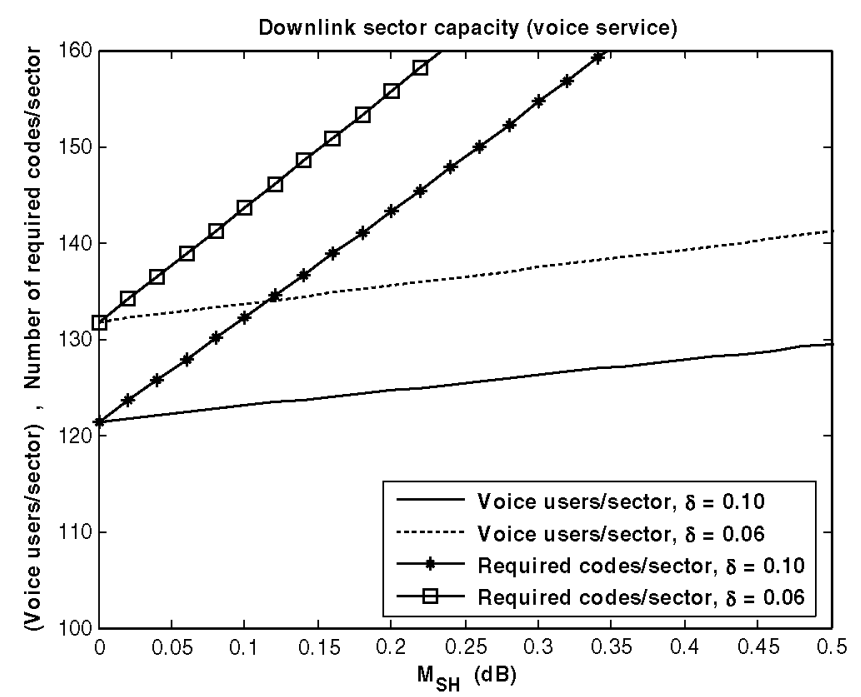

Fig. 9. Worst case sector downlink capacity and the required codes/sector as a function of $M_{\mathrm{SH}}$ for two different values of the orthogonality factor $\delta$.

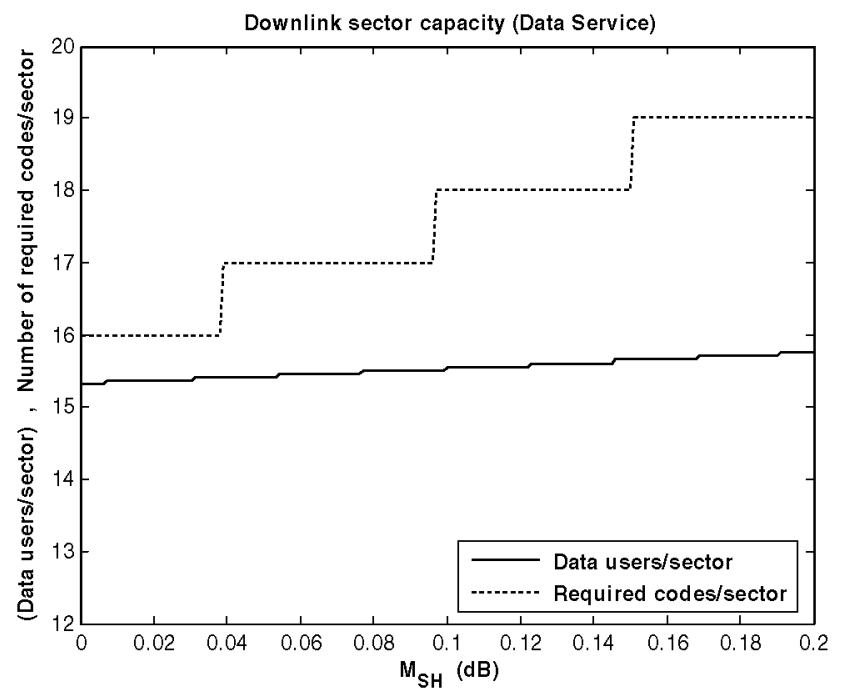

Fig. 10. Worst case sector downlink capacity and the required codes/ sector as a function of $M_{\mathrm{SH}}$ for the standard parameters considering the data service.

Finally, the case of data users will be studied assuming $\alpha=1, G_{\mathrm{p}}=32$ and $\left(E_{\mathrm{b}} / N_{\mathrm{o}}\right)_{\text {req }}=5.5 \mathrm{~dB}$. Fig. 10 portrays the sector downlink capacity as a function of $M_{\mathrm{SH}}$. It can be noticed that for $M_{\mathrm{SH}}$ of 0.038 , the sector downlink capacity is $\mathbf{1 5 . 4}$ data users and that the required number of codes is 16 codes.

The assigned power for the data users should be $\approx 5.26$ times the power assigned to the voice users. Fig. 11 represents the worst case sector downlink capacity for mixed services (voice and data).

For the worst case, the number of the users that can be supported within the train is the double of the sector downlink capacity.

We have to mention that, the best case downlink theoretical capacity of the sector is 618 voice users or 72 data

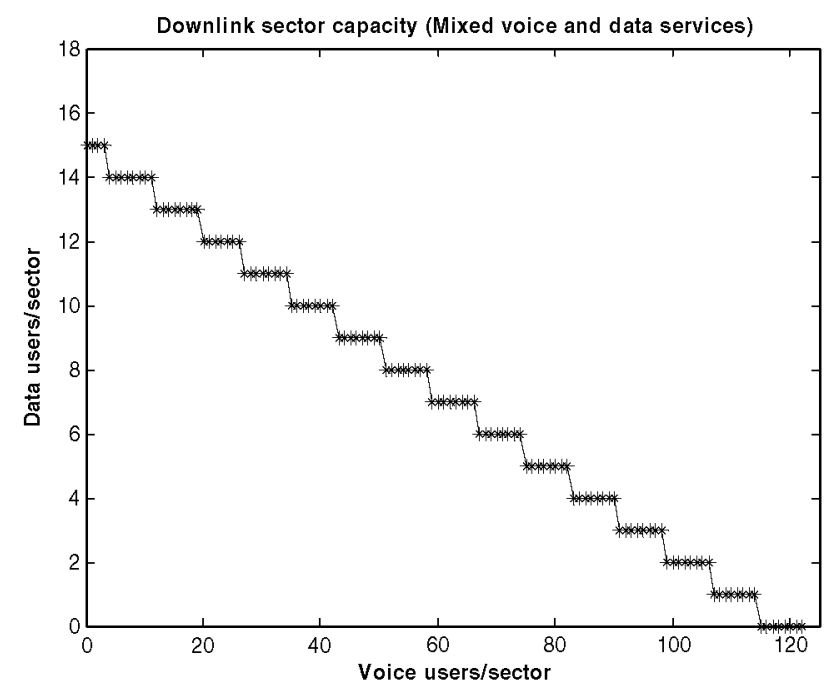

Fig. 11. Worst case sector downlink capacity (mixed voice and data services). 


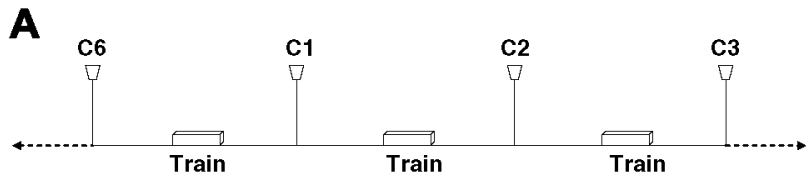

B

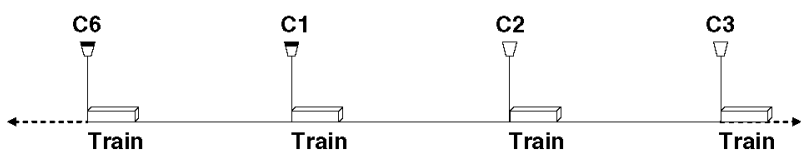

Fig. 12. Base stations and trains locations for the worst case and the best one. (A) The worst case. (B) The best case.

users. Also we have to mention that, the perfect power control can increase the sector capacity little bit and that the capacity increment factor for the worst case has an upper limit of $f_{\mathrm{c}}=\left(1+\frac{L}{4 R}\right)^{s_{2}}$. For the practical imperfect power control the capacity increment will be very small or even it can be negative (lower capacity using the practical imperfect power control in comparison with case when the power control is not used. For the best case, the capacity increment factor due to the perfect power control is quasi one $(1+\Delta)$ where $\Delta<<1$.

Fig. 12 depicts the base stations and trains locations for the worst case and the best one.

\section{Conclusions}

In this paper, the downlink performance of a cigarshaped microcells using wideband code-division multipleaccess (WCDMA) with soft hand-over (SHO) mode has been analyzed where microcells are deployed to give UMTS service to an over-ground train users. The twoslope propagation loss with log-normal shadowing has been used in the analysis where a model of eight cigarshaped microcells has been utilized to calculate the downlink sector capacity. The downlink sector capacity has been studied for different sector radius $R$, standard deviation of the shadowing $\left(\sigma_{1}\right.$ and $\sigma_{2}$ ) and propagation exponents $s_{1}$ and $s_{2}$. It has been found that, for a sector range higher than $940 \mathrm{~m}$, increasing the sector range will reduce the downlink sector capacity. Also it has been found that increasing the value of the propagation parameters $\left(s_{1}, s_{2}\right.$, $\sigma_{1}$ and $\sigma_{2}$ ) will reduce the downlink sector capacity. It has been found that, in many cases, the downlink sector capacity will be code limited since the theoretical downlink sector capacity is higher than the number of codes assigned to each sector. The high theoretical downlink sector capacity is due to the low value $(0.06-0.1)$ of the orthogonality factor in the highways microcells environment.

\section{References}

H. Holma, A. Toskala, WCDMA for UMTS, in: Radio Access for Third Generation Mobile Communications, Wiley, New York, 2001.
D. Wong, T.J. Lim, Soft handoffs in CDMA mobile systems, IEEE Pers. Commun. 4 (6) (1997) 6-17.

Radio Resource Control (RRC), Protocol Specification, 3GPP TSG RAN 25.331 V.5.2.0, 2002.

A.J. Viterbi, A.M. Viterbi, K.S. Gilhousen, E. Zehavi, Soft handoff extends CDMA cell coverage and increases reverse link capacity, IEEE J. Sel. Areas Commun. 12 (8) (1994) 1281-1288.

C. Mehailescu, X. Lagrange, P. Godlewski, Soft hand-over analysis in downlink UMTS WCDMA system, in: Proc. IEEE MoMuC, San Diego, CA, 1999, pp. 279-285.

X. Yang, S. Ghaheri-Niri, R.G. Tafazolli, Downlink soft hand-over gain in CDMA cellular network with cross-correlated shadowing, in Proc. IEEE Veh. Technol. Conf. VTC, Oct. 2001, vol. 1, pp. 276-280. D. Zhang, G. Wei, J. Zhu, Performance of hard and soft hand-over for CDMA system, in: Proc. IEEE Veh. Technol. Conf. VTC, Oct. 2002, vol. 2, pp. 1143-1147.

Y. Chen, L. Cuthbert, Optimum size of soft hand-over zone in power controlled UMTS downlink systems, Electron. Lett. 38(2)(2002) 89-90. B. Taha-Ahmed, M. Calvo-Ramon, L. Haro-Ariet, Capacity and interference statistics of highways W-CDMA cigar-shaped microcells (uplink analysis), IEEE Com. Lett. 6 (5) (2002) 172-174.

B. Taha-Ahmed, M. Calvo-Ramón, L. Haro-Ariet, On the uplink capacity and interference statistics of cigar-shaped road microcells in over-ground train service, 4th IASTED International Multi-conference Wireless and Optical Communications, Bnaff, Canada, 2004, pp. $109-114$.

C.C. Lee, R. Steele, CDMA for City Street Microcells, IEE Colloquium on Spread Spectrum Techniques for Radio Communication Systems, April 1993, pp. 3/1-3/10.

Ywh-Ren Tsai, Jin-Fu Chang, Feasibility of adding a personal communications network to an existing fixed-service microwave system, IEEE Trans. Com. 44 (1) (1996) 76-83.

Seungwook Min, Henry L. Bertoni, Effect of path loss model on CDMA system design for highway microcells, 48th VTC, Ottawa, Canada, May 1998, pp. 1009-1013. 